\title{
Multiple-unit activity of the rabbit cerebral cortex during stimulus generalization of avoidance behavior*
}

\author{
MICHAEL GABRIEL $\uparrow$, WILLIAM WHEELER $\uparrow \div$, and RICHARD F. THOMPSON $\uparrow \div$ \\ Department of Psychobiolog.', Lniversity of California, Inine, California 92664
}

\begin{abstract}
Multiple-unit neuronal activity was recorded from three "nonspecific" polysensory regions of the cerebral cortex of rabbit during a test for stimulus generalization of an avoidance CR. Behavioral and cortical data obtained during the acquisition of the CR were reported in a previous paper. These data showed acquisition and maintenance of cortical multiple-unit responses in two of the three cortical areas. paralleling acquisition and maintenance of the CR. The present results show significant behavioral gradients of stimulus generalization to manipulation of frequency of the tonal CS used in conditioning. In addition, significant generalization gradients based upon cortical multiple-unit responses to the CS and test stimuli were obtained from one of the two cortical areas showing an acquisition effect. The peaks of behavioral and cortical gradients were invariably associated with the CS frequency in different groups of rabbits trained with different CS frequencies. Thus, a "prewiring" interpretation of the tonal effects was ruled out. Our data indicate that the two characteristics of acquired stimulus control (acquisition and stimulus specificity) commonly observed for behavioral conditioned responses can be demonstrated using neuronal responses of the CNS. Further, such effects appear to occur within limited regions of the brain.
\end{abstract}

A previous paper (Gabriel, Wheeler, \& Thompson, 1973) outlined a potentially fruitful approach to the analysis of associative learning processes of the CNS, based upon the two general characteristics of associative learning that are shown in behavioral demonstrations of acquired stimulus control: (a) a change in response over conditioning trials (acquisition) and (b) acquired specificity of response to the training stimulus. Accordingly, we suggested that a neuronal response that develops during behavioral conditioning and shows specificity to the training stimulus represents a correlate of an associative process within the CNS. The specificity of a neuronal response to the training stimulus may be demonstrated in the same manner as behavioral specificity, by showing a significant stimulus generalization gradient with peak responsiveness to the CS used for conditioning.

In the previous paper (Gabriel et al, 1973), data were presented showing acquisition of cortical multiple-unit responses paralleling acquisition of a behavioral avoidance response to a pure-tone $\mathrm{CS}$. In the present

\footnotetext{
*Supported in part by Research Grant NS07661 from the National Institutes of Health (R.F.T.), Research Grant MH19314 from the National Institute of Mental Health (R.F.T.). Research Scientist Award MH06650 from the National Institute of Mental Health (R.F.T.). Postdoctoral Fellowship MH44158 from the National Institute of Mental Health (M.G.). and National Institute of Mental Health Training Grant MH11095 (W.W.). We wish to thank Shirley J. Adams and Fe E. Glanzman for technical assistance. We would like to express special appreciation to Richard Roemer, who provided the computer programs and other computer expertise essential for the analysis of the electrophysiological results of this and of our previous paper.

$\div$ Current address: Department of Psychology. Lniversity of Texas at Austin. Austin, Texas 78712. Requests for reprints should be sent to this address.

$\div \div$ Current address: Department of Psychology, Hariard University. Cambridge. Massachusetts 02138.
}

experiments, the behavioral and neuronal responses acquired through avoidance conditioning were tested for stimulus generalization to tones of different frequencies.

A critical issue for this study concerns the possibility that the neuronal response to one training frequency (e.g., $4 \mathrm{kHz}$ ) may be greater than that to other frequencies, independent of learning. To control for this, different groups of rabbits were trained with different $\mathrm{CS}$ frequencies. It was required that peak responsivness during a generalization test be associated with the frequency used as the CS in conditioning. In other words, both neuronal and behavioral generalization gradients must show "plasticity of peak."

Accordingly, generalization data are reported for three groups of Ss, each group given acquisition to a different CS frequency $(2,4$, or $8 \mathrm{kHz})$. Acquisition data for one of these groups (trained to a $4-\mathrm{kHz}$ CS) were reported in the previous paper (Gabriel et al, 1973).

\section{METHOD}

The basic procedure involved the recording of multiple-unit activity from three "nonspecific" polysensory cortical areas of the rabbit during stimulus generalization of avoidance behavior. We designated these three areas as anterior nonspecific (AN). dorsal nonspecific (DN), and posterior nonspecific (PN) (see Gabriel, Wheeler, \& Thompson, 1973). The location of the areas is shown in the left half of Fig. 1.

The details of the conditioning, recording, and data analysis are presented in Gabriel et al (1973).

Forty-one naive male albino rabbits received avoidance training in a single session on a day prior to the generalization test day. Training was given in a rotating-wheel conditioning apparatus (Brogden \& Culler, 1936). The CS and generalization test stimuli were pure tones of $5 \mathrm{sec}$ duration and $90 \mathrm{~dB}$ (re: $.0002 \mathrm{dynes} / \mathrm{cm}^{2}$ ) in intensity. A $4-\mathrm{kHz}$ tone served as the $\mathrm{CS}$ for 36 rabbits. Three rabbits were trained with a $2-\mathrm{kHz} \mathrm{CS}$, and two rabbits were trained with an $8 \mathrm{kHz}$ CS. During conditioning, the tone was followed by a $1-\mathrm{sec}$ shock. whereas generalization testing was carried out using an extinction (no shock) procedure. A rotational response to the tone (avoidance $C R$ ) served to abort 


\section{ELECTRODE \\ TARGETS}

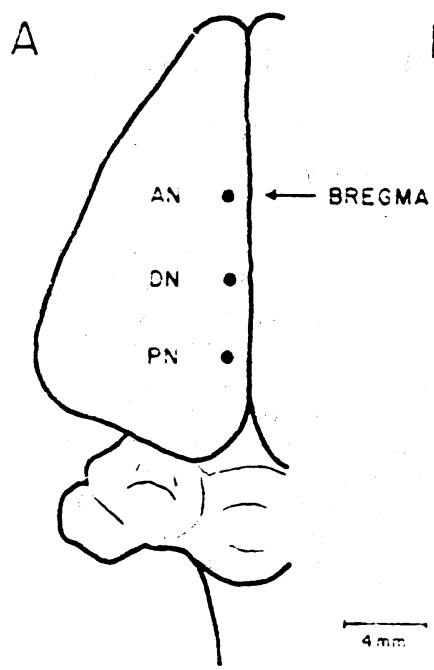

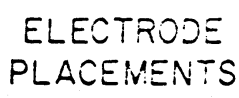

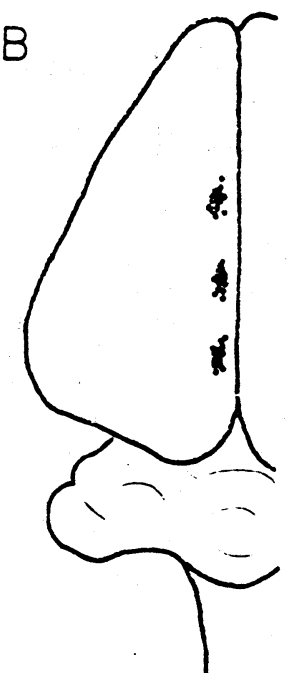

Fig. 1. Location of cortical target areas (A) and the surface topography of electrode placements (B). The left portion of this figure (A) shows the location of the three nonspecific cortical target areas within a diagram of the left cerebral hemisphere of the rabbit. The target areas are labeled $A N, D N$, and $P N$ for anterior, dorsal, and posterior nonspecific cortex, respectively. The right portion shows a scatter diagram of the actual electrode positions for the rabbits used in the electrophysiological analysis.

the scheduled shock in conditioning and to terminate the tone in both conditioning and generalization testing. The interval between tones was $10,15.20$. or $25 \mathrm{sec}$. These intervals occurred in a varied order.

Multiple-unit activity was recorded from chronically implanted, insulated stainless steel electrodes (15-20-micron tip diam. 50-100 microns exposed, $\sim 700 \mathrm{k}$ ohm impedance at $1 \mathrm{kHz}$ ) and was amplified using low-noise preamplifiers with field-effect transistor inputs. Data were continuously recorded on magnetic tape during acquisition and generalization testing.

\section{Experimental Design}

The generalization test was conducted using tones which differed in frequency, but had the same intensity $(90 \mathrm{~dB})$ as the training CS. The intensity of the CS had been systematically varied during the overtraining phase of conditioning to control for possible perceptual intensity differences that may have been associated with the various frequencies used in the present generalization test.

Each of the rabbits trained with a $4-\mathrm{kHz}$ CS was returned to the apparatus either $24 \quad \mathrm{~N}=18)$ or $48(\mathrm{~N}=18) \mathrm{h}$ after acquisition. Half of the $\mathrm{Ss}$ in each delay condition received 20 trials with the CS $(4 \mathrm{kHz})$ followed by 20 trials with a $1-\mathrm{kHz}$ generalization tone. The other half received a 20 -trial block (Block 1) with the generalization tone followed by 20 trials with the CS (Block 2). After the initial 40 test trials, each $S$ received presentations of $1,2,8$, and $16 \mathrm{kHz}$ tones in blocks of 5 consecutive trials. All Ss received four separate 5-trial blocks of each generalization tone for a total of 20 trials with each tone. The order of tone blocks was determined by using a 4 by 4 Latin square, and each $S$ progressed through the entire Latin square ( 80 trials). After the Latin square, each $S$ received 20 additional trials with the CS $(4 \mathrm{kHz})$. A block diagram of the experimental design is shown in Fig. 2.

Rabbits trained with a $2-\mathrm{kHz} C S \quad \mathrm{~N}=3$ ) and an $8-\mathrm{kHz} \mathrm{CS}$

$(\mathrm{N}=2)$ received generalization testing $24 \mathrm{~h}$ after conditioning. using a similar procedure. However. the first 20 trials of extinction (Block 1) were with the generalization tone $(8 \mathrm{kHz}$ for animals trained with $2 \mathrm{kHz}$, and $2 \mathrm{kHz}$ for rabbits trained with $8 \mathrm{kHz}$ ), and the second 20 trials were with the CS. Also. for these Ss. the 2- and $8-\mathrm{kHz}$ CSs were given in the final 20 post-Latin-square trials and $4 \mathrm{kHz}$ replaced 2 or $8 \mathrm{kHz}$ as a Latin-square generalization tone.

\section{Data Analysis}

As in the previous study (Gabriel et al, 1973). behavioral data are reported for all Ss. Ten rabbits from the group of 36 trained with a $4-\mathrm{kHz}$ CS were selected for analysis of electrophysiological data. The $10 \mathrm{Ss}$ selected were those showing large-amplitude multiple-unit records ( $50 \mathrm{microV}$ peak-to-peak minimum) in all three cortical areas (AN, DN, and PN). Six of these rabbits received the $\mathrm{CS}$ for the first 20-trial block of extinction followed by 20 trials with the $1-\mathrm{kHz}$ generalization tone, and four received the stimuli in the opposite order. The records of all five rabbits trained to a $2-$ or $8-\mathrm{kHz}$ CS met the electrophysiological criterion for data anlysis and were therefore used to obtain both behavioral and electrophysiological data.

Multiple-unit data were reported only for electrode tips shown to lie within the cortical gray matter. The histological selection resulted in use of 9 out of $10 \mathrm{AN}$ implants, 10 of $10 \mathrm{DN}$ implants, and 8 of 10 PN implants within the 10 rabbits trained with a $4-\mathrm{kHz}$ CS. All AN, DN, and PN implants of the five rabbits trained with a 2 - or $8-\mathrm{kHz} \mathrm{CS}$ fell within the cortical gray matter and were used for data analysis. The right half of $\mathrm{Fig.} 1$ shows a scatter plot of the locations of these electrodes.

The tapes of the multiple-unit records of the selected Ss were played back through a high-pass filter and a notch filter into. a Schmidt trigger. The notch filter was set to the frequency of the training CS. The uniform, discrete output pulses from the Schmidt trigger were totalled by a PDP-12 computer (Digital Equipment Corporation) in consecutive 4-msec time "bins" preceding and following tone onset. Time histograms were compiled for blocks of trials by cumulating counts over consecutive trials in corresponding bins.

Two 20-trial histograms based on the initial 40 extinction trials were compiled for each electrode of each animal. One of these reflected $20 \mathrm{CS}$ trials, and the other reflected 20 generalization trials. A separate 5-trial histogram was compiled using the last five CS presentations preceding the Latin square.

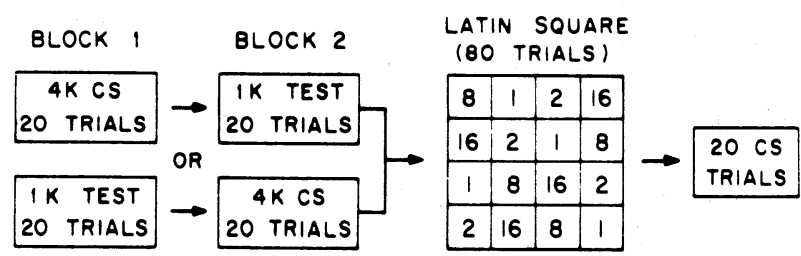

Fig. 2. Block diagram of the experimental design used for the generalization (extinction) test. Half of the rabbits progressed through Block 1 and Block 2 receiving the $4-\mathrm{kHz}$ CS followed by the $1-\mathrm{kHz}$ generalization tone. This is illustrated by the upper two left-hand blocks of the diagram. The remaining half received the stimuli in the opposite order. This is illustrated by the lower two left-hand blocks of the diagram. All rabbits then progressed through the entire Latin square (center part of diagram). Each rabbit was assigned to a starting row of the Latin square so that the starting row factor was equally weighted within the remaining conditions of the experiment. Once a starting row was determined for a given rabbit, the remaining rows followed in the order shown in the diagram. All rabbits received 20 trials with the CS after the square (right part of diagram). 

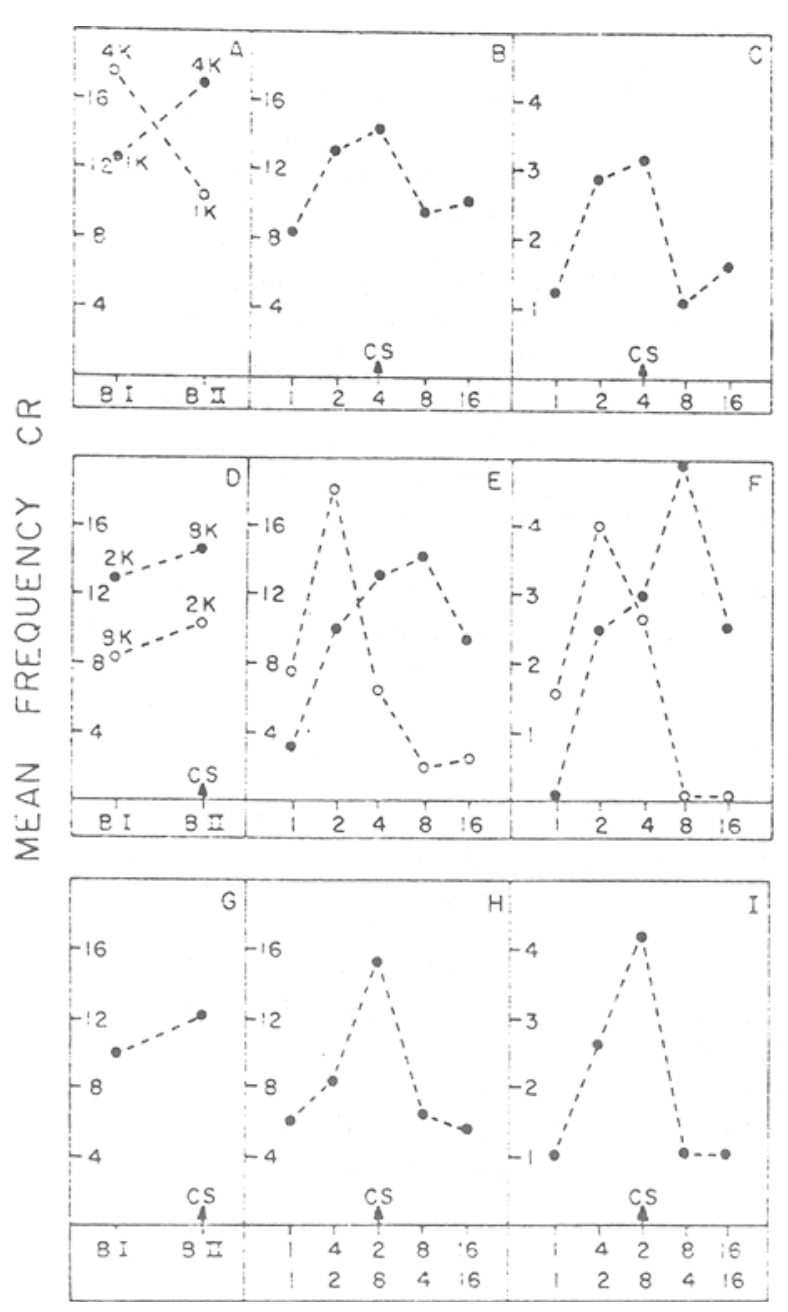

Fig. 3. Average behavioral gradients of stimulus generalization for the three phases of the generalization (extinction) test. The mean frequency of $C R$ is plotted for each of the tone frequencies $(\mathrm{kHz})$ of the test. In all plots, the upward-pointing arrows indicate the frequency of the CS. Parts A, B, and C show the data of the 36 rabbits trained with a $4-\mathrm{kHz}$ CS. In Part A, the open circles represent the data of 18 of the rabbits given 20 presentations of the $4-\mathrm{kHz}$ CS in Block 1 (BI), followed by 20 presentations of the $1-\mathrm{kHz}$ test tone, Block 2 (BII) of the test. The filled circles represent the opposite order of tone presentation experienced by the other 18 rabbits. Part B shows the gradient based on performance of 36 rabbits during the Latin square. The CS data of this gradient were obtained from trials prior to and following the square (see main text for details). The gradient of Part $C$ was based on the performance of 36 rabbits during the final Latin square row and the first five postsquare CS presentations. Parts D. E. and F show the average gradients of three rabbits trained with a $2 \cdot \mathrm{kHz} \mathrm{CS}$ (open circles) and of two rabbits trained with an $8-\mathrm{kHz}$ CS (filled circles). For the purposes of statistical analysis, data from the rabbits trained to a $2-\mathrm{kHz} \mathrm{CS}$ and those trained to an $8-\mathrm{kHz}$ CS were combined by (a) computing the mean frequency of CR for the two groups in Blocks 1 and 2, (b) ordering the Latin square data of each group with the CS frequency located centrally $(1,4,2,8$, and $16 \mathrm{kHz}$ for group trained with $2-\mathrm{kHz}$ CS: $1,3.8,4$, and $16 \mathrm{kHz}$ for group trained with $8-\mathrm{kHz} \mathrm{CS}$ ). and (c) computing the mean frequency of $C R$ associated with each pair of tones resulting from superimposition of the reordered Latin square scales $[1+1.4+2,2+8$ (CSs). $8+4.16+16 \mathrm{kHz}]$. The pooled data of these two groups are shown in G. H. and I.
Sixteen 5-trial histograms were compiled for the Latin square, one for each of the 16 tone blocks. Additionally, four 5-trial histograms were compiled from the final $20 \mathrm{CS}$ presentations given after the Latin square.

Three numerical scores (cortical t scores) were computed for each histogram (see Gabriel et al, 1973). The $t$ score was based on the mean number of counts in each of three consecutive 5-bin $(20-\mathrm{msec})$ periods following tone onset. Each of the three post-tone-onset means was subtracted from a comparable pretone mean, and the difference was divided by the standard error of the mean difference. The cortical $t$ score expresses the magnitude and direction of change in multiple-unit activity following a stimulus in standard units of ongoing variation. An individual cortical $t$ score may be referred to the Student $t$ distribution with the appropriate degrees of freedom $(8$ in the present study) in order to determine a probability level for the multiple-unit activity of the $20-\mathrm{msec}$ posttone periods. A 60 -msec analysis period was used for the present generalization data instead of the $200-\mathrm{msec}$ period used for the data of conditioning, because the significant changes in multiple-unit activity during conditioning occurred in the first $50 \mathrm{msec}$ following CS onset (Gabriel et al, 1973).

\section{RESULTS}

Two general findings were obtained in the present study: (1) Significant behavioral stimulus generalization gradients to tone frequency were found. The data of Fig. 3 show peak behavioral responsivness to each of three different tones used as CSs in this study. The behavioral gradients for the animals trained to a $4-\mathrm{kHz}$ CS are shown in Figs. 3A, 3B, and 3C. The gradients of the group trained with a $2-\mathrm{kHz} \mathrm{CS}$ and the group trained with an $8 \mathrm{kHz}$ CS are shown in Figs. 3D, 3E, and $3 \mathrm{~F}$. (2) Significant generalization gradients were obtained using cortical multiple-unit responses of the dorsal nonspecific area (DN). Peak multiple-unit responsivness in DN was associated with the tone frequency used as the CS. The other cortical areas (AN and PN) did not yield significant neuronal gradients. Figure 4 shows the neuronal gradients from $\mathrm{AN}, \mathrm{DN}$, and $\mathrm{PN}$ of the group trained to a $4-\mathrm{kHz} \mathrm{CS}$. Figs. $5 \mathrm{~A}, 5 \mathrm{~B}$, and $5 \mathrm{C}$ show the neuronal gradients (DN only) of the rabbits trained to a $2 \cdot$ and $8 \cdot \mathrm{kHz} \mathrm{CS}$.

The records of individual rabbits (Figs. 6 and 7) further illustrate these findings. A more detailed description of the results is given below.

\section{Behavioral CRs, All Ss}

Figures $3 \mathrm{~A}, 3 \mathrm{~B}$, and $3 \mathrm{C}$ show the group behavioral stimulus generalization gradients to tone frequency for all 36 rabbits trained to a $4-\mathrm{kHz}$ CS. The gradients shown in Fig. $3 \mathrm{~A}$ were based upon the number of CRs in Blocks 1 and 2 of extinction. Half of the Ss conditioned with a $4-\mathrm{kHz} \mathrm{CS}$ received 20 sequential presentations of the CS followed by 20 presentations of a $1 \cdot \mathrm{kHz}$ generalization tone (circles). The other half of the Ss received the two tones in the opposite order (filled circles). A 2 by 2 factorial repeated measures analysis of variance (ANOVA), with order of tones and 20-trial test blocks as the main factors, was performed on these data. $\mathrm{A}$ significant $\mathrm{F}$ for the Order by Blocks interaction $(\mathrm{F}=$ 


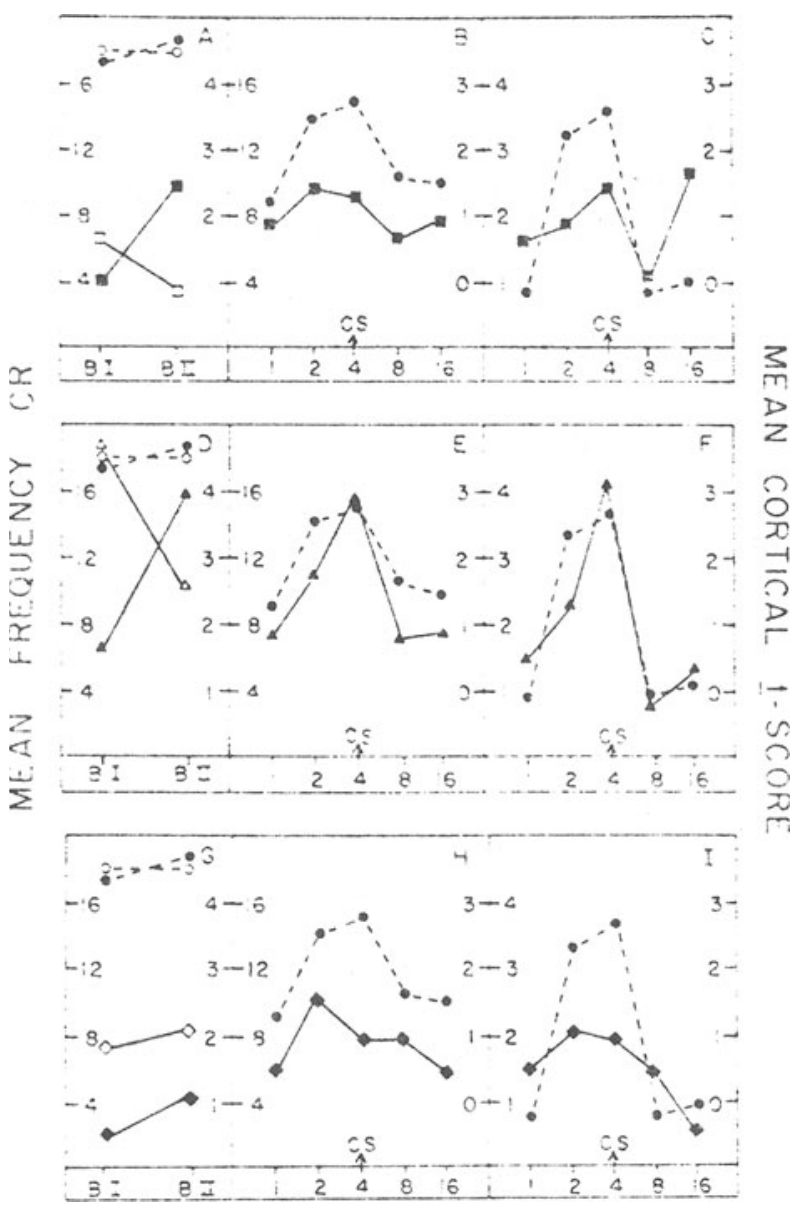

Fig. 4. Average cortical and behavioral gradients of stimulus generalization for rabbits trained with a $4-\mathrm{kHz}$ CS. These gradients were obtained from the subset of rabbits $(N=10$ ) selected for electrophysiological analysis. The solid lines represent the mean cortical $t$ score averaged over the initial three 20 -msec post-CS onset periods (right ordinates). The dotted lines represent behavioral gradients based on the mean frequency of CR (left ordinates). The gradients of the three horizontal subsections were obtained from Blocks 1 and 2 of the test, the overall Latin square, and the final Latin square row, respectively. The three vertical subsections represent, from top to bottom, the three cortical areas (A-C from AN, D-F from DN, G-I from PN). The behavioral gradients are plotted redundantly within each of the vertical subsections to facilitate comparison of behavioral and cortical data. In Parts A, D. and G, the open symbols represent the data of Ss given the 4-kHz CS in Block 1 (20 trials) followed by the $1-\mathrm{kHz}$ test tone in Block 2 ( 20 trials). The filled $5 y$ mbols represent data of the Ss given the opposite order of tones. In all plots. the upward-pointing arrows indicate the frequency of the CS.

29.97. $\mathrm{df}=1 / 34, \mathrm{p}<.001$ ) was obtained. Post-ANOVA comparisons of CS vs test-tone responses (two-tailed t test. $\propto=.05$ ) showed a significantly greater mean frequency of $\mathrm{CR}$ to the $4-\mathrm{kHz} \mathrm{CS}$ compared to the $1-\mathrm{kHz}$ generalization tone for both orders of tone presentation. Thus, a significant behavioral generalization decrement was obtained in the initial phase of the test for the rabbits trained to a $4-\mathrm{kHz} \mathrm{CS}$.
The intersession delay factor did not produce a significant $\mathrm{F}$ in this or any other analysis of the study.

The gradient of Fig. $3 \mathrm{~B}$ was based on the responses to four generalization stimuli $(1.2 .8$. and $16 \mathrm{kHz})$ of the Latin square. Since the CS was not presented in the Latin square, CS data for this gradient were obtained from CS trials preceding and following the square. These control trials were obtained in the following manner. The five final CS trials preceding the square and the first 15 trials following the square were used. The 5 presquare trials were used as control data for the first row of the square. The 15 postsquare trials were divided into three 5-trial blocks, which were assigned to Rows 2, 3, and 4 of the Latin square, respectively. A 5 by 4 factorial repeated measures ANOVA was performed using tone frequency $(1,2,4,8$, and $16 \mathrm{kHz})$ and Latin square rows (four levels) as factors. The main effect of tone frequency was significant $(F=11.25$, df $=4 / 420$, $\mathrm{p}<.001)$, and post-ANOVA comparisons showed a significantly greater mean frequency of $\mathrm{CR}$ to the $4-\mathrm{kHz}$ CS than to all four generalization stimuli. Additionally, a significant effect of Latin square rows was obtained $(\mathrm{F}=$ 34.71, df $=3 / 105, p<.001)$. indicating overall extinction of behavioral CRs. The means of the four successive Latin square rows were: 3.522 . 3.161.2.427. and 2.005 .

The gradient shown in Fig. 3C represents data of the final row of the Latin square and the initial 5 postsquare CS trials. A one-way repeated measures ANOVA showed

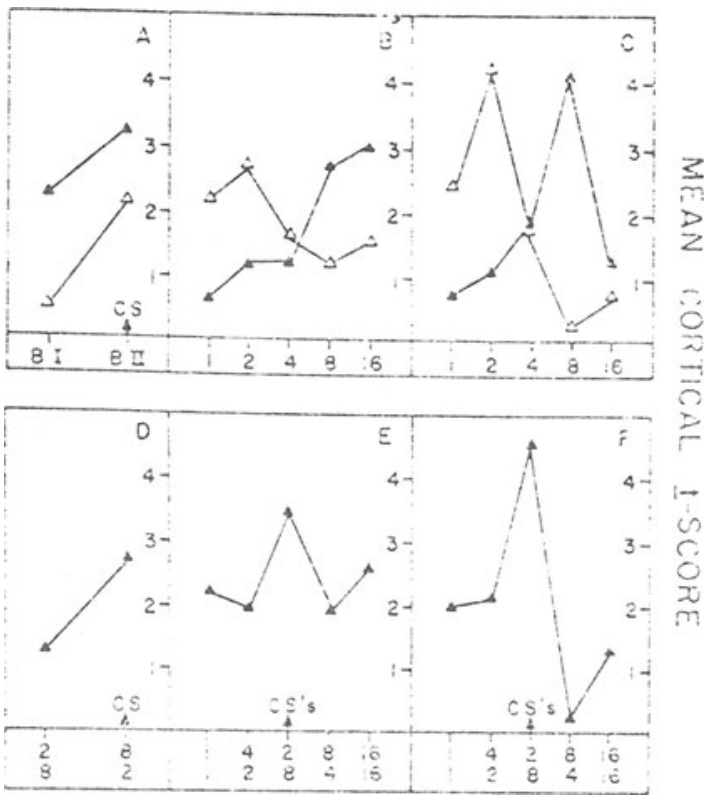

Fig. 5. Average generalization gradients based on cortical $t$ scores of $\mathrm{DN}$ for the rabbits trained with a $2 \cdot \mathrm{kHz} \mathrm{CS}(\mathrm{N}=3)$ or an $8-\mathrm{kHz}$ CS $(\mathrm{N}=2)$. Parts $\mathrm{A}$. B. and $\mathrm{C}$ show the gradients of Blocks 1 and 2 . the entire Latin square, and the final Latin square row, respectively, for the $2-\mathrm{kHz}$ group (open triangles) and for the $8 \cdot \mathrm{kHz}$ group (filled triangles). Parts D. E. and F. show the gradients in each phase of the test obtained by pooling the multiple-unit data for the two groups (see caption. Fig. 3). 
$\neq 32$

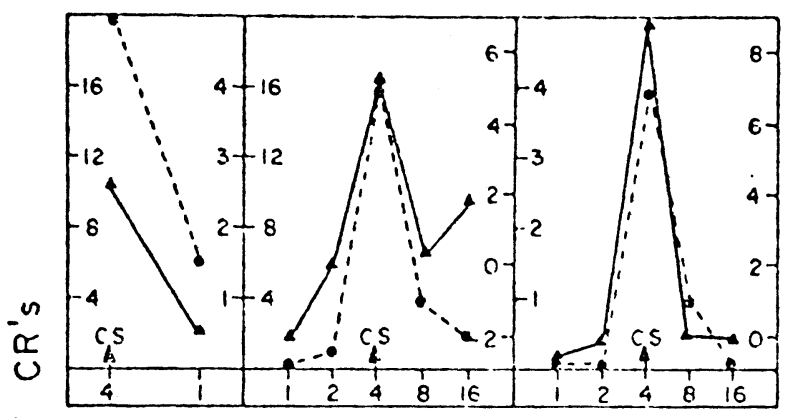

\#34

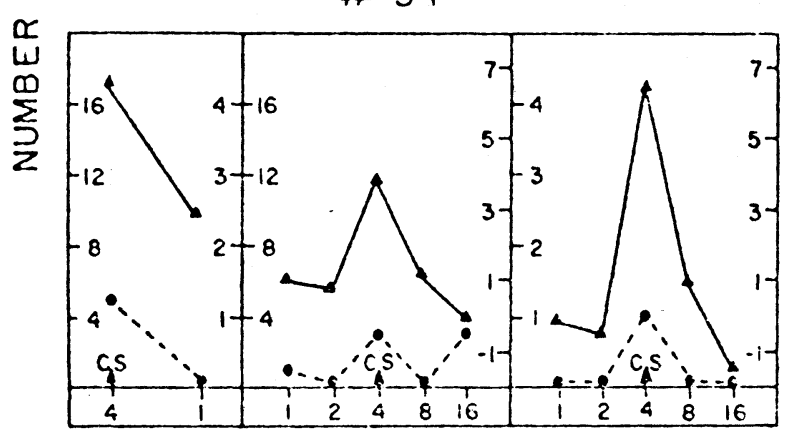

\# 3 !
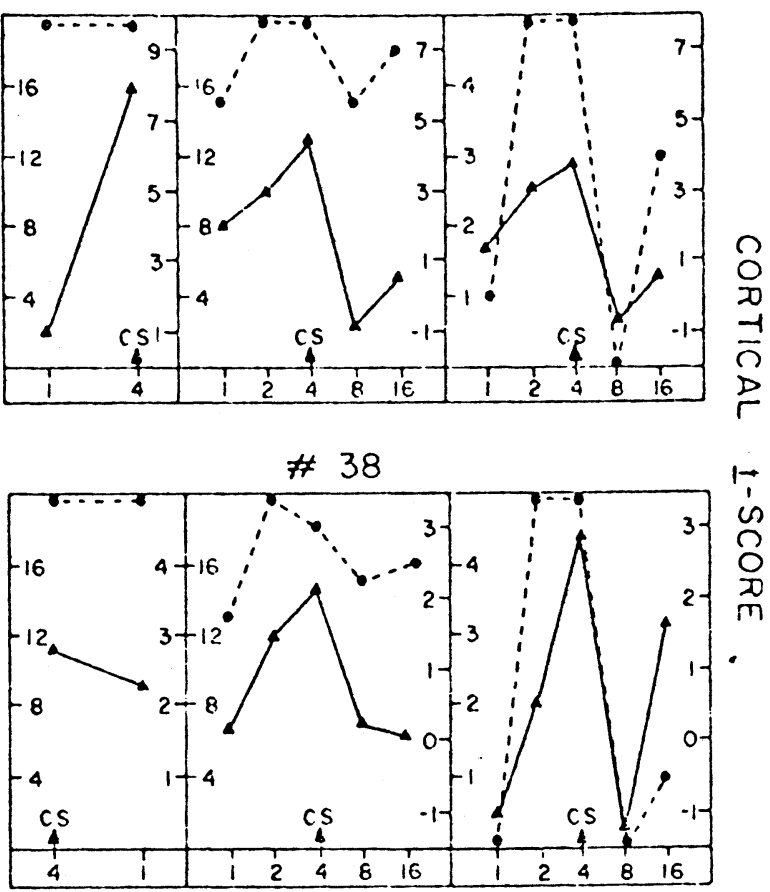

Fig. 6. Generalization gradients of four individual rabbits trained with a $4 \cdot \mathrm{kHz}$ CS (31, 32, 34, and 38) based on multiple-unit responses (cortical t scores) of DN (solid lines, right ordinates), and number of CRs (dotted lines, left ordinates). See previous figures for details of gradient construction.

a significant effect of tone frequency for this gradient $(F$ $=16.37$, df $=4 / 140 . p<.001)$, and post-ANOVA comparisons showed a significantly greater mean frequency of $C R$ to the $C S$ than three of the four

$\# 2 \mathrm{C}$

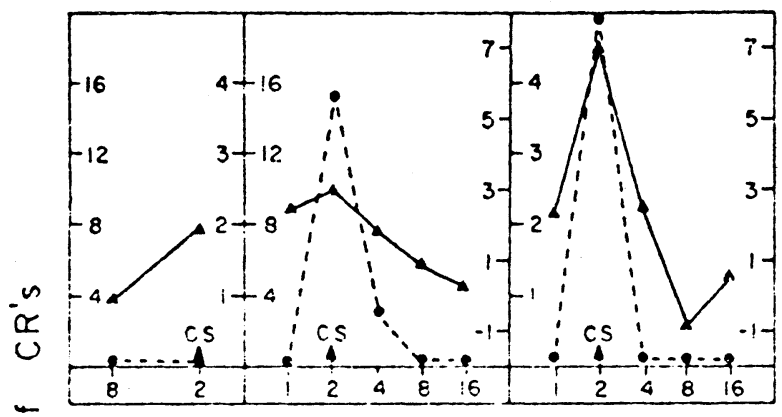

$\div$

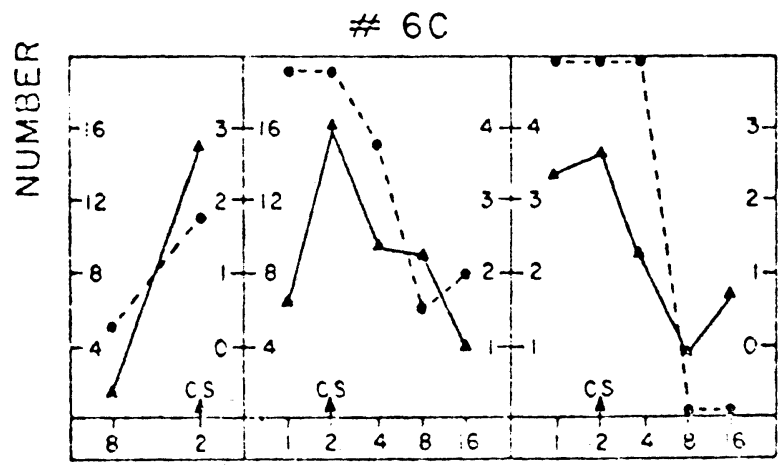

generalization stimuli. However, the numerical superiority of the CS mean over the $2-\mathrm{kHz}$ mean was not significant. The method of constructing the gradients shown in Figs. 3A, 3B, and 3C applies essentially to all

$\#$ IC

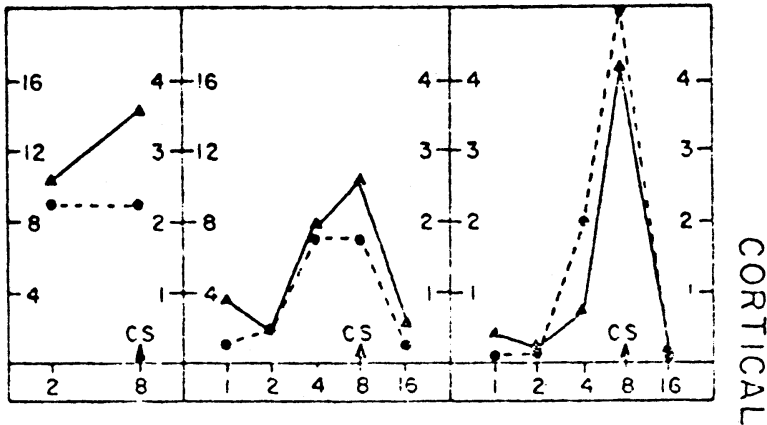

$\# 3 \mathrm{C}$

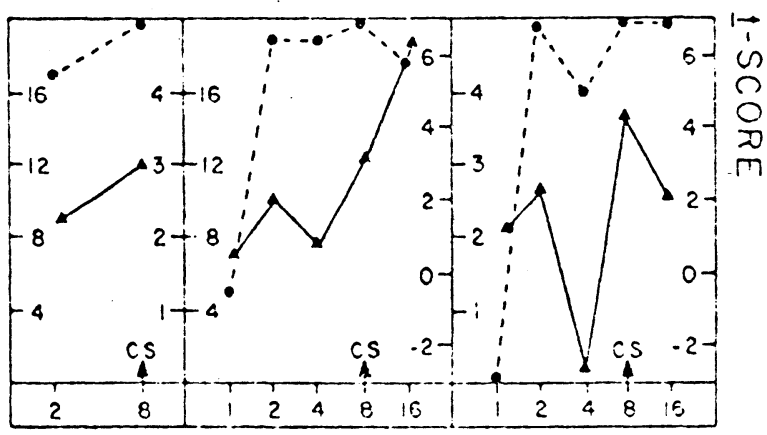

Fig. 7. Ceneralization gradients of four individual rabbits trained with a $2-\mathrm{kHz} C \mathrm{CS}(2 \mathrm{C}$ and $6 \mathrm{C})$ or an $8 \cdot \mathrm{kHz} \mathrm{CS}(1 \mathrm{C}$ and $3 \mathrm{C})$ based on multiple-unit responses (cortical t scores) of DN (solid lines. right ordinates). and number of CRs (dotted lines. left ordinates). See previous figures for details of gradient construction. 
gradients described below.

Figures $3 \mathrm{D} .3 \mathrm{E}$. and $3 \mathrm{~F}$ show the behavioral gradients of rabbits trained with a $2 \cdot \mathrm{kHz} \mathrm{CS}(\mathrm{N}=3$. circles $)$ and an $8 \mathrm{kHz}$ CS $(\mathrm{N}=2$. filled circles $)$. For these Ss. the first 20 trials were given with the generalization tone, and the second 20 trials were given with the CS. The generalization tone was $8 \mathrm{kHz}$ for rabbits trained with $2 \mathrm{kHz}$. and $2 \mathrm{kHz}$ for those trained with $8 \mathrm{kHz}$. In both groups. the $4-\mathrm{kHz}$ tone was used in the Latin square instead of the CS tone. The CS tone was presented as usual for 20 trials following the Latin square.

The data of Ss trained with 2 - and $8 \cdot \mathrm{kHz}$ CSs were pooled for the statistical analysis. The gradients based on the pooled data are shown in Figs. 3G, 3H. and 3I. Note in Figs. 3H and 3I that the tone frequencies are arranged so as to locate the CS frequency in the center position.

The data of Blocks 1 and 2 (Fig. 3G) and of the overall Latin square (Fig. $3 \mathrm{H}$ ) showed a greater mean frequency of $\mathrm{CR}$ to the CS compared to the test tones. However, the tone frequency factor did not produce significant $F$ ratios for these gradients. A significant $F$ for tone frequency was obtained for the gradient based on the final Latin square row (Fig. $3 \mathrm{I} ; \mathrm{F}=5.56$, df $=$ $4 / 16, p<.005$ ), and post-ANOVA comparisons showed a significantly greater mean frequency of $\mathrm{CR}$ to the $\mathrm{CS}$ than to each of the test frequencies.

In summary, the data portrayed in Fig. 3 show peak behavioral responsiveness to each of three different tones used as CSs in this study. Thus, "innate" differential effectiveness of particular tones in eliciting the behavioral response may be ruled out.

\section{Cortical Multiple-Unit Responses}

The previous study (Gabriel et al, 1973), using a $4-\mathrm{kHz}$ CS, showed that no CS-evoked activity was detectable in AN. DN, or PN in the initial trials of conditioning. As CS-UCS pairing progressed, CS-evoked multiple-unit responses developed in AN and DN. These responses showed a significant increase in magnitude over conditioning trials paralleling the behavioral acquisition function. This effect occurred within the initial 50-msec period following CS onset, and the multiple-unit response had an onset latency of $15.25 \mathrm{msec}$. In addition, the $\mathrm{AN}$ and $\mathrm{DN}$ responses were consistently present while the rabbits performed at asymptotic levels during overtraining. No acquisition-related alterations in multiple-unit activity from PN were detected. Although not reported previously, the conditioning results of Ss trained with a 2. or $8 \cdot \mathrm{kHz}$ CS were essentially the same as the $4 \cdot \mathrm{kHz}$ results.

The data described in this section utilized the cortical $t$ scores of three consecutive $20-\mathrm{msec}$ post-CS onset periods in order to evaluate generalization of acquired multiple-unit responses.

Figure 4 shows the mean cortical t scores averaged over the three $20-\mathrm{msec}$ post-CS onset periods and across
10 Ss trained with a 4-kHz CS (right ordinates, solid lines). The scores are plotted in the same format used for the behavioral gradients. Since the post-CS onset periods factor did not produce significant results. all plots of cortical $t$ scores represent averages over the three periods. Figures 4A. 4B and 4C show gradients based on AN t scores, Figs. 4D, 4E, and $4 \mathrm{~F}$ show DN t scores, and Figs. 4G, 4H, and $4 \mathrm{I}$ show the $\mathrm{PN} t$ scores. The tone frequency factor did not produce significant $F s$ for either AN or PN scores. However, tone frequency did produce significant Fs when DN scores were used. The analysis of Blocks 1 and 2 (DN data) showed a significant Order by Blocks interaction $(F=6.10 . \mathrm{df}=$ $1 / 6, p<.01)$. reflecting the significant "crossover" of the two cortical gradients of Fig. 4D. These findings indicated that a greater mean cortical $t$ score was associated with the $4-\mathrm{kHz} \mathrm{Cs}$ compared to the $1 \cdot \mathrm{kHz}$ generalization tone. This difference held for both orders (4-1 and 1-4) of tone presentation.

Analyses of cortical $t$ scores of DN for the overall Latin square (Fig. 4E) and for the final row of the square (Fig. 4F) yielded findings similar to those of Blocks 1 and 2. A significant tone frequency effect $[F($ overall square $)=4.88, \mathrm{df}=4 / 36, \mathrm{p}<.01$. and $\mathrm{F}($ final row $)=4.27, \mathrm{df}=4 / 36, \mathrm{p}<.01]$ was obtained for the $\mathrm{t}$ scores of DN only. Post-ANOVA comparisons showed a significantly greater mean cortical $t$ score to be associated with the $4-\mathrm{kHz}$ CS compared to the 8- and $16-\mathrm{kHz}$ means in both gradients. The remaining comparisons were not significant in either of the gradients. Additionally, the effect of Latin square rows was not significant for the overall gradient, suggesting absence of an overall extinction effect in the cortical data.

The behavioral generalization gradients of the subset of 10 rabbits selected for electrophysiological analysis are plotted redundantly in each of the three vertical subsections in Fig. 4 (dashed lines, left ordinates). The gradients of the entire square $[F=4.87, \mathrm{df}=4 / 36$, $\mathrm{p}<.001$ (Figs. 4B, 4E, and 4H)] and the final Latin square row $[F=8.87, d=4 / 36, p<.001$ (Figs. $4 C, 4 F$, and $4 \mathrm{I}$ )] were significant. and post-ANOVA comparisons for both gradients showed a significantly greater mean frequency of $C R$ to the $C S$ relative to all other means except the $2-\mathrm{kHz}$ mean. In addition. the overall extinction effect obtained for the behavioral data of the large group $(\mathrm{N}=36)$ was also obtained for the subset of 10.

Figure 5 shows the generalization gradients based on the cortical $t$ scores of $\mathrm{DN}$ in rabbits trained with a 2- or $8 \mathrm{kHz}$ CS. In Figs. 5A, 5B, and 5C, the average cortical gradients are shown separately for the 2- and $8 \cdot \mathrm{kHz}$ groups, and may be compared to the behavioral gradients of the same groups plotted in Figs. 3D, 3E. and $3 \mathrm{~F}$. In Figs. 5D, 5E, and 5F, the $2-$ and $8 \cdot \mathrm{kHz}$ gradients are pooled, as done previously for the behavioral gradients (Figs. 3G, 3H, and 3I). Although analyses of Block 1 and Block 2 data and of the overall Latin square 
data did not show significant effects, the analysis of the pooled gradient of the last Latin square row (Fig. 5F) showed a significant effect of tone frequency $(F=3.91$, $\mathrm{df}=4 / 8, \mathrm{p}<.025)$, and post-ANOVA comparisons showed mean cortical responsiveness associated with the CS to be significantly greater than the means of the other gradient points. These data suggest that significant superiority of the DN cortical responses to the CS does not depend upon use of a particular CS frequency, but occurs for a variety of different CS frequencies. Thus, plasticity of gradient peak appears to be a property of the acquired neuronal responses of DN. There were no significant $F s$ in the analyses of AN and PN data for rabbits trained with the 2- or $8-\mathrm{kHz}$ CSs.

The neuronal and behavioral data of Figs. 6 and 7 show generalization gradients of individual rabbits. Figure 6 shows gradients from four rabbits trained to a $4-\mathrm{kHz}$ CS. Figure 7 shows the records of two rabbits trained to a $2-\mathrm{kHz} \mathrm{CS}$ (left of figure) and two rabbits trained to an $8 \cdot \mathrm{kHz}$ CS (right of figure). All of the individual DN gradients except one (bottom right, Fig. 7) show a numerical peak associated with the CS frequency, indicating that the significant group gradients presented above were not artifacts of the procedures involved in obtaining average gradients.

\section{DISCUSSION}

Our experiments were designed to detect changes in neuronal activity within the CNS resulting from behavioral conditioning. In addition, we hoped that such changes would reflect associative processes of the nervous system rather than nonassociative correlates of learning, such as arousal. In order to approach the distinction between associative and nonassociative neural events experimentally, we required that neuronal responses show the same properties commonly held to reflect "associativeness" in other classes of responses (e.g., skeletal or autonomic). Therefore, we focused on conditioned neuronal responses meeting the twofold criterion of stimulus control: (1) a change over conditioning trials, and (2) specificity of acquired response to the training stimulus.

Data obtained from the present study and our previous study (Gabriel et al, 1973) indicate that the neuronal responses of DN satisfy the twofold criterion. Specifically, neuronal responses of this region, not present on initial conditioning trials, came to be evoked by the avoidance CS on later trials of conditioning within a single training session. This acquisition followed a function parallel to the acquisition of the behavioral $\mathrm{CR}$, and both neuronal and behavioral responses showed maintenance during 100 postcriterial trials (overtraining) of conditioning. The same finding was observed for the acquisition records of AN. These data represented fulfillment of the initial (acquisition) criterion of acquired stimulus control. The second criterion of stimulus control, requiring the demonstration of statistically reliable generalization gradients, was also fulfilled by behavioral data and by neuronal data of DN. Significant generalization gradients were demonstrated on a test day following conditioning, using an extinction procedure in which the same tone frequency used as the CS, as well as different generalization frequencies, were presented. Data from different groups of Ss, each trained with a different CS frequency, showed generalization gradients with peak behavioral and DN neuronal responsiveness at the frequency used as the CS. Thus, both behavioral and neuronal (DN) generalization gradients showed the property of gradient peak plasticity, which rules out a "prewiring" interpretation of gradient peaks.

It is interesting to note that the other cortical area (AN) which showed acquisition and maintenance of neuronal responses during conditioning did not show significant neuronal gradients. This finding, together with the absence of evidence for either acquisition or stimulus specificity in PN, suggests that the neuronal stimulus control effects may occur only within limited regions of the brain. Findings consistent with this viewpoint have been reported by Olds and his associates (Olds et al, 1972; Segal \& Olds, 1972; Segal, Disterholt, \& Olds, 1972).

Consideration of an anatomically distinct stimulus control system raises interesting questions regarding alternative ways in which such a system may function. For example, one may inquire about the level of "generality" of the acquired stimulus coding that is reflected in the responses of DN. Stimulus specificity is a very general property of conditioned behavior. Given any particular stimulus as a CS, behavioral gradients will occur to manipulation of several, if not all, of the dimensions of the stimulus (see Terrace, 1967). Thus, symmetrical gradients may be produced by postacquisitional manipulation of frequency or intensity of a tonal stimulus, or by manipulation of wavelength, saturation, or intensity of a photic stimulus. Behavioral control seems to be predicated on "change" of a stimulus in a very general sense, although stimulus properties differ in terms of the relative ease of establishing control (Mackintosh, 1965; Terrace, 1967). At any rate, it is of interest to inquire whether neuronal responses showing acquired stimulus control will show the same level of generality as that shown by behavioral responses.

An alternative possibility would be that different regions show stimulus control to different properties of stimuli. On this hypothesis, DN might be regarded as a neuronal analyzer for acquired auditory frequency discrimination. Other regions may be analyzers for different dimensions and modalities of stimuli. Indeed, separate analyzers for different stimulus properties have been predicated on the basis of behavioral studies of animal discrimination learning (e.g.. Mackintosh, 1965; Sutherland \& Mackintosh, 1971).

A final point of interest has to do with the 
observations of discrepancy between our neuronal and our behavioral data. For example, the subset of 10 rabbits trained to a $4-\mathrm{kHz} \mathrm{CS}$ showed a performance "ceiling" effect. i.e.. maximal frequency of CR to both CS and test stimulus in the initial phase (Blocks 1 and 2) of the test. Thus. reliable behavioral generalization gradients were not obtained for the subset in the initial test phase. However. the neuronal (DN) gradients of the subset were statistically reliable in all three test phases. Could it be that the neuronal response is not subject to performance factors (such as ceiling effects) characteristic of behavioral CRs? An additional finding supportive of this viewpoint was the absence of evidence for an overall decrease (extinction) of the neuronal response over test trials in the present experiment, although highly significant extinction of CR performance did occur.

These instances of neuronal-behavioral discrepancy suggest that neuronal data may provide a more direct and sensitive index of acquired stimulus control than that provided by traditional behavioral measures. It may be that neuronal data will vield results approximating theoretical conceptions of learning, such as that embodied in Hull's concept of habit strength (Hull, 1943). more closely than do behavioral responses. It may be possible eventually to achieve a "mapping" of the mammalian central nervous system which delineates separate anatomical systems yielding strictly performance-correlated activity and systems yielding more purely "learning-related" activity. Ultimately. some resolution of the problem of performance in the study of learning might be achieved with this approach.

\section{REFERENCES}

Brogden. W. J., \& Culler, E. A. Device for motor conditioning of small animals. Science, 1936, 83. 269.

Gabriel. M.. Wheeler. W., \& Thompson, R. F. Multiple-unit activity of the rabbit cerebral cortex in single-session avoidance conditioning. Phỵsiological Psychology. 1973. 1. 45-55.

Hull. C. L. Principles of behavior: An introduction to behavior theory: New York: Appleton-Century-Crofts, 1943.

Mackintosh, N. J. Selective attention in animal discrimination learning. Psychological Bulletin, 1965. 64, 124-150.

Olds. J.. Disterhoft. J.. Segal. M., Kornblith, D. L., \& Hirst. R. Learning centers of rat brain mapped by measuring latencies of conditioned unit responses. Journal of Neurophysiology. 1972. 35. 202-219.

Segal. M.. Disterhoft, J., \& Olds. J. Hippocampal unit activity. during classical aversive and appetitive conditioning. Science, $1972,175,792-794$.

Segal. M.. \& Olds, J. Behavior of units in hippocampal circuit of rat during learning. Journal of Neurophysiology. 1972. 35. 680-690.

Sutherland. N. S., \& Mackintosh. N. J. Mechanisms of animal discrimination learning. New York: Academic Press. 1971.

Terrace. H. S. Stimulus control. In W. K. Honig (Ed.). Operant behavior areas of research and application. New York: Appleton-Century-Crofts. 1966.

(Received for publication June 15. 1973: accepted July 26. 1973.) 